\title{
Long non-coding RNA ferritin heavy polypeptide 1 pseudogene 3 controls glioma cell proliferation and apoptosis via regulation of the microRNA-224-5p/tumor protein D52 axis
}

\author{
YONGQIANG ZHANG ${ }^{1}$, YING LI $^{2}$, JING WANG $^{2}$ and PING LEI ${ }^{1}$ \\ ${ }^{1}$ Geriatric Ward of Neurology, Department of Geriatrics, Institute of Tianjin Geriatrics; \\ ${ }^{2}$ Tianjin Key Laboratory of Lung Cancer Metastasis and Tumor Microenvironment, Tianjin Lung Cancer Institute, \\ Tianjin Medical University General Hospital, Tianjin 300052, P.R. China
}

Received November 14, 2017; Accepted July 25, 2018

DOI: $10.3892 / \mathrm{mmr} .2018 .9491$

\begin{abstract}
The aim of the present study was to investigate the potential role and regulatory mechanism of long non-coding RNA ferritin heavy polypeptide 1 pseudogene 3 (FTH1P3) in glioma development. The expression of FTH1P3 in low- and high-grade glioma tissues was investigated using reverse transcription-quantitative polymerase chain reaction. FTH1P3 expression was overexpressed or suppressed in U251 glioma cells to examine the involvement of FTH1P3 in glioma cell proliferation and apoptosis using MTT assay and flow cytometry respectively. In addition, the regulatory association between FTH1P3, microRNA (miR)-224-5p and tumor protein (TP) D52 was investigated to elucidate the potential underlying mechanisms of FTH1P3 in glioma by luciferase reporter assay. The results revealed that FTH1P3 was up-regulated in glioma tissues, and FTH1P3 expression in high-grade glioma tissues was significantly higher compared with that in low-grade glioma tissues. Upregulation of FTH1P3 promoted glioma cell proliferation and inhibited apoptosis. Furthermore, FTH1P3 inhibited miR-224-5p expression, which in turn negatively regulated TPD52 expression. Overexpression of miR-224-5p significantly inhibited U251 cell proliferation and induced cellular apoptosis; this effect was clearly reversed following co-transfection of miR-224-5p and TPD52. These data revealed that upregulation of FTH1P3 may have promoted glioma cell proliferation and inhibited apoptosis. Thus, the miR-224-5p/TPD52 axis may be a downstream mechanism of FTH1P3 in glioma progression. The findings of the present
\end{abstract}

Correspondence to: Professor Yongqiang Zhang or Professor Ping Lei, Geriatric Ward of Neurology, Department of Geriatrics, Institute of Tianjin Geriatrics, Tianjin Medical University General Hospital, 154 Anshan Road, Tianjin 300052, P.R. China E-mail: yongqiangzhang06@126.com

E-mail: ytaklv@sina.com

Key words: glioma, long non-coding RNA ferritin heavy polypeptide 1 pseudogene 3 , microRNA-224-5p, tumor protein D52 study may provide a theoretical basis for the study of the treatment of glioma in the future.

\section{Introduction}

Glioma is an aggressive subtype of primary brain tumor with an extremely poor prognosis (1), in which glioblastoma is the most severe manifestation. It is reported that only 3-5\% patients with glioblastoma survive for $>5$ years following diagnosis (2). Owing to the highly aggressive and invasive nature of glioblastoma, current therapeutic strategies, including neurosurgery, radiation therapy and chemotherapy, are ineffective (3). Thus, it is important to elucidate the key mechanisms underlying glioma development and progression.

Long non-coding RNAs (lncRNAs) are RNA molecules that are not translated into proteins. They have gained increasing attention due to their diverse functions in various physiological and pathological processes (4-6). The dysregulation of lncRNAs has been demonstrated to have specific roles in a variety of cancer types, including bladder, prostate and kidney cancer (7-9). Furthermore, a number of lncRNAs may be promising biomarkers or targets for the diagnosis and treatment of cancer including breast and lung cancer $(10,11)$. In glioma, numerous lncRNAs, including metastasis associated lung adenocarcinoma transcript 1 (12), HOXA11 antisense RNA (13), X-inactive specific transcript (14) and H19 imprinted maternally expressed transcript (15), function as key mediators in tumor development. Recently, lncRNA ferritin heavy polypeptide 1 pseudogene 3 (FTH1P3), a member of the FHC gene family, has been demonstrated to contribute to the progression of oral squamous cell carcinoma and uveal melanoma by functioning as a miR-224-5p sponge $(16,17)$. However, to the best of our knowledge, whether IncRNA FTH1P3 expression is dysregulated and involved in glioma development by sequestering miR-224-5p has not yet been reported.

In the present study, the expression of lncRNA FTH1P3 in low- and high-grade glioma tissues was detected. Following this, FTH1P3 was overexpressed and suppressed in glioma $\mathrm{U} 251$ cells in vitro to investigate its roles in regulating cell proliferation and apoptosis. As discussed above, FTH1P3 functioned as an important regulator in the development 
and progression of oral squamous cell carcinoma and uveal melanoma by sequestering miR-224-5p $(16,17)$, and miR-224 inhibits the development of prostate cancer by targeting tumor protein D52 (TPD52) (18). Therefore, the regulatory association between FTH1P3 and the miR-224-5p/TPD52 axis was investigated in glioma cells to further elucidate the potential regulatory mechanism of FTH1P3. The findings of the present study may provide a broader perspective for the treatment of this disease.

\section{Materials and methods}

Tissue samples. Between March 2016 and April 2017, 20 patients (aged $27.3 \pm 9.6$, male:female $=12: 8$ ) with glioma were admitted to Tianjin Medical University General Hospital (Tianjin, China). Fresh high- and low-grade glioma samples (6 cases were high-grade and 14 were low-grade) and adjacent normal tissues were obtained from these patients during a temporal lobectomy procedure for epilepsy and immediately snap-frozen in liquid nitrogen upon surgical removal. The present study was approved by the ethics committee of Tianjin Medical University General Hospital according to the criteria of the Declaration of Helsinki. Informed consent was acquired from each patient.

Cell culture and transfection. The human glioma cell line U251 was purchased from the European Collection of Cell Cultures (09063001; ECACC, Porton Down, Salisbury, UK; deposited by Shanghaisixin Biotech Co., Ltd, Shanghai, China) and maintained in Dulbecco's modified Eagle's medium (Gibco; Thermo Fisher Scientific, Inc., Waltham, MA, USA) with $10 \%$ fetal bovine serum (Hyclone; GE Healthcare Life Sciences, Logan, UT, USA), $100 \mu \mathrm{g} / \mathrm{ml}$ streptomycin and $100 \mathrm{U} / \mathrm{ml}$ penicillin in a $5 \% \mathrm{CO}_{2}$ incubator at $37^{\circ} \mathrm{C}$.

$\mathrm{U} 251$ cells of an appropriate concentration (approximately $80 \%$ ) at $1 \times 10^{5}$ were seeded on 6 -well plates and were incubated at $37^{\circ} \mathrm{C}$ in the plates for a further $24 \mathrm{~h}$ prior to transfection. Cells were subsequently transfected with appropriate concentrations (200 $\mu \mathrm{M}$ for each) of pcDNA3.1-FTH1P3 (pc-FTH1P3) (Sangon Biotech Co., Ltd., Shanghai, China), small interfering RNA (siRNA)-FTH1P3, miR-224-5p mimic, pcDNA3.1-TPD52 (pc-TPD52) and the corresponding controls using Lipofectamine ${ }^{\circledR} 2000$ reagent (Invitrogen; Thermo Fisher Scientific, Inc.). Medium was replaced following $4 \mathrm{~h}$ of transfection. The sequences for the target vectors were: FTH1P3 siRNA, 5'-CACCGCCAG CCCTCCGTCACCTCTTCGAAAAGAGGTGACGGAGGG CTGGC-3'; miR-224-5p mimic sense, 5'-CAAGUCACUAGU GGUUCCGUU-3' and antisense, 5'-CGGAACCACUAGUGA CUUGUU-3'; siRNAcontrol sense, 5'-CACCGGGAGAAT GCGATGGGAGAGCCGAAGCTCTCCCATCGCATTCTC CC-3' and antisense: 5'-AAAAGGGAGAATGCGATGGGA GAGCTTCGGCTCTCCCATCGCATTCTCCC-3'; miRNA mimic control, sense 5'-AAAAUGGUGGUGCCCUAG UGACUACA-3' and antisense, 5'-UAGUCACCAUAAUAG GGCACCAUUUUUU-3'. Cells were harvested for further experimentation at 24,48 and $72 \mathrm{~h}$ following incubation.

MTT assay. Cell viability was detected with a MTT assay. Transfected cells were seeded in a 96-well plate at a density of $2 \times 10^{4}$ cells/well at $37^{\circ} \mathrm{C}$ and allowed to attach overnight. MTT solution $(20 \mu \mathrm{l} ; 0.5 \mathrm{mg} / \mathrm{ml}$; Sigma-Aldrich; Merck KGaA, Darmstadt, Germany) was subsequently added to each well for $4 \mathrm{~h}$ at $37^{\circ} \mathrm{C}$. Medium was removed and $0.2 \mathrm{ml}$ dimethyl sulfoxide was added to each well for $30 \mathrm{~min}$ at $37^{\circ} \mathrm{C}$. The optical density of each well at $490 \mathrm{~nm}$ was read on an enzyme-labeled instrument (microplate reader, Bio-Rad Laboratories, Inc., Hercules, CA, USA) to assess cell viability.

5-bromo-2-deoxyuridine (BrdU) incorporation assay. Following transfection, cells at a density of $1 \times 10^{5}$ cells/well were incubated with $10 \mu \mathrm{M}$ BrdU (Sigma-Aldrich; Merck $\mathrm{KGaA}$ ) at $37^{\circ} \mathrm{C}$ for $48 \mathrm{~h}$ and fixed in $4 \%$ paraformaldehyde at $37^{\circ} \mathrm{C}$ for $30 \mathrm{~min}$. Cells were subsequently incubated with $0.1 \%$ Triton $\mathrm{X}-100$ for $10 \mathrm{~min}, 2 \mathrm{M} \mathrm{HCl}$ for $5 \mathrm{~min}$ and $0.1 \mathrm{M}$ borate buffer ( $\mathrm{pH} 8.5$ ) for $5 \mathrm{~min}$ in the proper order, as listed, at $37^{\circ} \mathrm{C}$. To detect the BrdU-positive cells, cells were blocked with $2 \%$ bovine serum albumin (FBS; Gibco; Thermo Fisher Scientific, Inc.), probed with an anti-BrdU antibody (1:1,000; ab1893; Abcam, Cambridge, UK) overnight at $4^{\circ} \mathrm{C}$, followed by incubation with a fluorescein isothiocyanate (FITC)-labeled anti-mouse immunoglobulin $\mathrm{G}$ secondary antibody (1:1,000; ab157532; Abcam). The percentage of cells with positive staining was examined in three randomly selected high-power fields at x400 magnification using a fluorescence microscope and Cellquest software (version 3.0; BD Biosciences, Franklin Lakes, NJ USA).

Flow cytometry detection. The apoptosis rate of each group was examined with an Annexin V-FITC apoptosis detection kit (Beijing Biosea Biotechnology Co., Ltd, Beijing, China), according to the manufacturer's protocol. Briefly, cells $\left(5 \times 10^{6}\right)$ were harvested, seeded in a 6 -well plate and mixed with $10 \mu \mathrm{l}$ Annexin and $5 \mu \mathrm{l}$ propidium iodide $(10 \mathrm{mg} / \mathrm{l})$ for $15 \mathrm{~min}$ in the dark. Apoptosis was detected with a flow cytometer (BD Biosciences) and the apoptotic percentages were analyzed with BD CellQuest 3.0 software (BD Biosciences). Annexin V-positive cells were regarded as apoptotic cells.

Luciferase reporter assay. TargetScanHuman version 7.1 (http://www.targetscan.org/vert_72/) was used to predict the target sequences of miR-224-5p in TPD52. To construct the wild-type (WT) luciferase reporter vectors, the TPD52 3'-untranslated region (3'-UTR), which included the miR-224-5p seed binding sites, was cloned into the pGL3-promotor vector (Sangon Biotech Co., Ltd., Shanghai, China). In the mutation-type (MUT) luciferase reporter vector, the predicted seed zones in miR-224-5p were replaced by nonsense sequences. For the luciferase reporter assay, $100 \mathrm{ng}$ TPD52 3'UTR luciferase construct and $400 \mathrm{ng}$ miR-224-5p mimics were co-transfected into U251 cells using Lipofectamine ${ }^{\circledR} 2000$ reagent (Invitrogen; Thermo Fisher Scientific, Inc.). The relative luciferase activity of the luciferase construct was subsequently detected using a Dual-Glo Luciferase assay kit (E2940; Promega Corporation, Madison, WI, USA) following $48 \mathrm{~h}$ transfection. Firefly luciferase activity was normalized to Renilla activity. The $\beta$-galactosidase expression vector was used as a control for transfection efficiency. 
Reverse transcription-quantitative polymerase chain reaction $(R T-q P C R)$. Total RNA was extracted from tissues and cells using TRIzol ${ }^{\circledR}$ reagent (Invitrogen; Thermo Fisher Scientific, Inc.) and cDNA was synthesized at $70^{\circ} \mathrm{C}$ using a Maxima First Strand cDNA Synthesis kit (Thermo Fisher Scientific, Inc.) according to the manufacturer's protocol [after heating to $94^{\circ} \mathrm{C}$ for $2 \mathrm{~min}$, the volume $(50 \mu \mathrm{l})$ was subjected to 30 cycles of $94^{\circ} \mathrm{C}$ for $30 \mathrm{sec}, 61^{\circ} \mathrm{C}$ for $30 \mathrm{sec}$, and $72^{\circ} \mathrm{C}$ for $30 \mathrm{sec}$. The gene expression levels of FTH1P3, miR-224-5p and TPD52 were determined by SYBR green qPCR kit (Thermo Fisher Scientific, Inc.) and calculated using the $2^{-\Delta \Delta C q}$ method (19). The primer sequences used were as follows: FTH1P3 forward, 5'-TCC ATTTACCTGTGCGTGGC-3' and reverse, 5'-GAAGGAAGA TTCGGCCACCT-3'; miR-224-5p forward, 5'-CTGGTAGGT AAGTCACTA-3' and reverse, 5'-TCAACTGGTGTCGTG GAG-3'; U6 forward, 5'-CTGGTAGGGTGCTCGCTTCGG CAG-3' and reverse, 5'-CAACTGGTGTCGTGGAGTCGG C-3'; TPD52 forward, 5'-CGTTGTATTTGTTATTTATCA AGTTGT-3' and reverse, 5'-TCCCCAAGTAAATCTAGTCAT GC3'; GAPDH forward, 5'-AAGACCTTGGGCTGGGAC TG-3' and reverse, 5'-ACCAAATCCGTTGACTCCGA-3'. PCR product was produced under the following parameters: 1 Predenaturation cycle of $2 \mathrm{~min}$ at $94^{\circ} \mathrm{C}, 32$ cycles of $95^{\circ} \mathrm{C}$ for $15 \mathrm{sec}, 64^{\circ} \mathrm{C}$ for $30 \mathrm{sec}$, and $72^{\circ} \mathrm{C}$ for $2 \mathrm{~min}$, with a final extension at $72^{\circ} \mathrm{C}$ for $5 \mathrm{~min}$. GAPDH was used as the endogenous control for FTH1P3 and TPD52, whereas U6 was used as an internal control for miR-224-5p expression.

Western blot analysis. Total protein was extracted from cells with radioimmunoprecipitation assay lysis buffer (Sangon Biotech Co., Ltd., Shanghai, China) and the concentration was measured with a bicinchoninic acid protein assay kit. Equal amounts of protein (50 $\mu \mathrm{g}$ per lane) were subjected to $10 \%$ SDS-PAGE and blotted onto polyvinylidene fluoride membranes. Membranes were blocked in Tris-buffered saline with Tween-20 containing 5\% non-fat milk at $4^{\circ} \mathrm{C}$ overnight, and subsequently incubated with primary rabbit monoclonal antibodies against apoptosis regulator Bcl-2 (BCL2; 1:1,000; cat. no. ab32124), apoptosis regulator BAX (BAX; 1:1,000; cat. no. ab32503), TPD52 (1:1,000; cat. no. ab181260) and GAPDH (1:1,000; cat. no. ab8245; Abcam, Cambridge, UK) overnight at $4^{\circ} \mathrm{C}$. Following this, the membrane was probed with horseradish peroxidase-conjugated goat anti-rabbit secondary antibody (1:5,000; cat. no. ab97051; Abcam) for $2 \mathrm{~h}$ at room temperature. An enhanced chemiluminescence kit (Santa Cruz Biotechnology, Inc., Dallas, TX, USA) was used to visualize the protein bands. Western blot quantification was performed using ImageJ v1.48 software (National Institutes of Health, Bethesda, MD, USA). GAPDH was used as the reference protein.

Statistical analysis. All experiments were repeated at least three times and the obtained data are presented as the mean \pm standard deviation. Student's t-tests were used for the analysis of statistical significance between two groups, and one-way analysis of variance followed by Dunnett's post hoc test was applied to analyze statistical significance among three groups or more. All statistical analysis was conducted using SPSS 13.0 software (SPSS, Inc., Chicago, IL, USA). P<0.05 was considered to indicate a statistically significant difference.

\section{Results}

LncRNA FTH1P3 is upregulated in glioma tissues, and its upregulation promotes the proliferation and inhibits the apoptosis of glioma cells. The expression of FTH1P3 in glioma tissues was initially determined. As presented in Fig. 1A, FTH1P3 expression in glioma tissues was significantly increased compared with that in normal tissues $(\mathrm{P}<0.001)$; furthermore, TH1P3 expression in high-grade glioma tissues was significantly higher compared with low-grade glioma tissues $(\mathrm{P}<0.05)$, indicating that the upregulation of FTH1P3 may be associated with the development and progression of glioma. Thus, FTH1P3 was overexpressed and suppressed in glioma U251 cells to investigate the potential role of FTH1P3 in glioma in vitro. Following transfection with pc-FTH1P3 and siRNA-FTH1P3, FTH1P3 expression was successfully overexpressed and suppressed, compared with transfection with the corresponding control $(\mathrm{P}<0.01$, FTH1P3 vs. blank control; $\mathrm{P}<0.001$ si-FTH1P3 vs. si-control; Fig. 1B). The results of the MTT assay demonstrated that overexpression of FTH1P3 markedly increased cell viability after 24, 48 and $72 \mathrm{~h}$, whereas suppression of FTH1P3 had the opposite effect ( $\mathrm{P}<0.05$ at $24 \mathrm{~h}, \mathrm{P}<0.01$ at 48 h, and $\mathrm{P}<0.001$ at 72 h; Fig. $1 \mathrm{C}$ ). Similar results were obtained from BrdU assay; BrdU-positive cells were significantly increased in the pc-FTH1P3 group compared with the blank group, whereas positive cells were markedly decreased in the siRNA-FTH1P3 group compared with the siRNA control group $(\mathrm{P}<0.05$, FTH1P3 vs. blank control; $\mathrm{P}<0.001$ si-FTH1P3 vs. si-control; Fig. 1D). The results of MTT and BrdU assays indicated that overexpression of FTH1P3 promoted glioma U251 cell proliferation. In addition, flow cytometry revealed that the percentage of apoptotic cells in the pc-FTH1P3 group was significantly lower compared with that of the blank group, whereas the percentage in the siRNA-FTH1P3 group was significantly increased compared with the siRNA-control group $(\mathrm{P}<0.01$, FTH1P3 vs. blank control; P<0.01 si-FTH1P3 vs. si-control; Fig. 1E). Notably, western blot analysis demonstrated that the alterations in BAX/BCL2 expression were in line with the percentage of apoptotic cells in each transfected group $(\mathrm{P}<0.05$, FTH1P3 vs. blank control; $\mathrm{P}<0.001$ si-FTH1P3 vs. si-control; Fig. 1F). These data indicated that overexpression of FTH1P3 may have inhibited glioma U251 cell apoptosis via regulation of BAX/BCL2 expression.

LncRNA FTH1P3 inhibits miR-224-5p expression. It has been reported that FTH1P3 facilitates the progression of oral squamous cell carcinoma by inhibiting miR-224-5p (16). Thus, the regulatory association between FTH1P3 and miR-224-5p in U251 cells was investigated. As presented in Fig. 2A, miR-224-5p expression was significantly decreased in glioma tissues compared with normal tissues $(\mathrm{P}<0.05)$; however, there was no significant difference in miR-224-5p expression between high- and low-grade glioma tissues. In addition, miR-224-5p expression was significantly decreased in the pc-FTH1P3 group and increased in the siRNA-FTH1P3 group, compared with the corresponding control groups $(\mathrm{P}<0.01$, FTH1P3 vs. blank control; $\mathrm{P}<0.05$ si-FTH1P3 vs. si-control; Fig. 2B). These data indicated that miR-224-5p expression was inhibited by FTH1P3 in glioma U251 cells. To further 

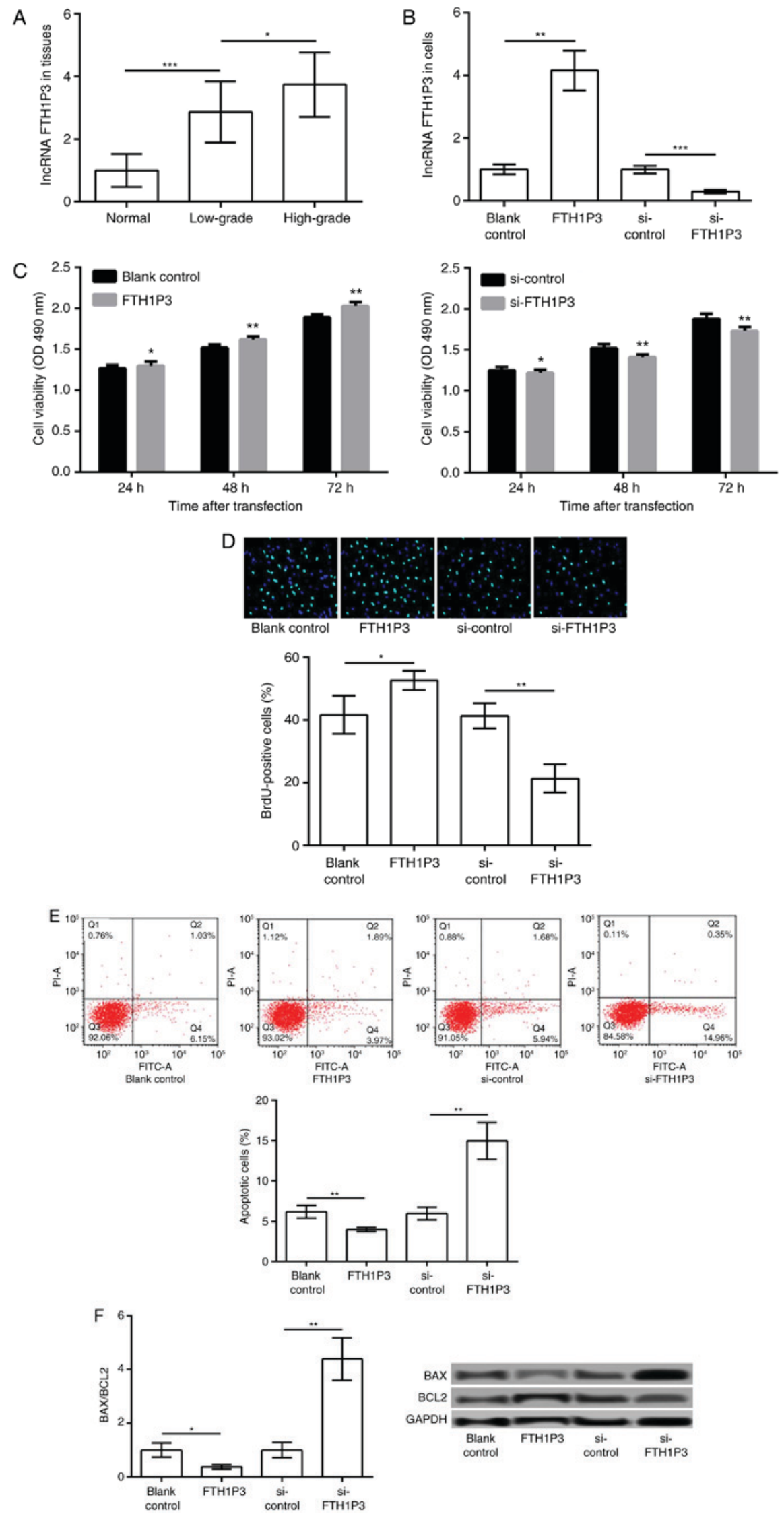

Figure 1. LncRNA FTH1P3 is upregulated in glioma tissues, and its upregulation promotes glioma cell proliferation and inhibits apoptosis. (A) The expression of lncRNA FTH1P3 in low- and high-grade glioma tissues and adjacent normal tissues. (B) Expression of lncRNA FTH1P3 in glioma U251 cells transfected with pc-FTH1P3, si-FTH1P3 and the corresponding controls. (C) The cell viability of each group after $24,48 \mathrm{~h}$ and $72 \mathrm{~h}$ was determined with an MTT assay. (D) A BrdU incorporation assay was performed to determine cell proliferation (magnification, $\mathrm{x} 400$ ). (E) The percentage of apoptotic cells in each group was determined by flow cytometry. (F) Western blot analysis of BAX/BCL2 expression. " $\mathrm{P}<0.05,{ }^{* *} \mathrm{P}<0.01$ and ${ }^{* * *} \mathrm{P}<0.001$. IncRNA, long non-coding RNA; FTH1P3, ferritin heavy polypeptide 1 pseudogene 3; pc, pcDNA3.1; si, small interfering; BrdU, 5-bromo-2-deoxyuridine; BAX, apoptosis regulator BAX; BCL2, B cell lymphoma 2; OD, optical density; FITC, fluorescein isothiocyanate. 
A

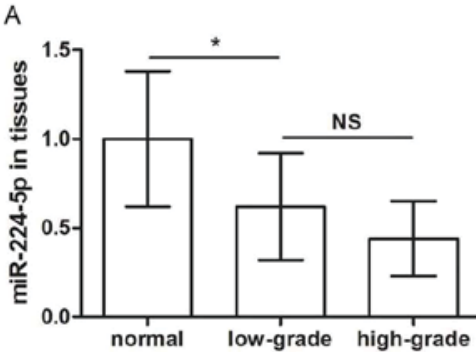

B

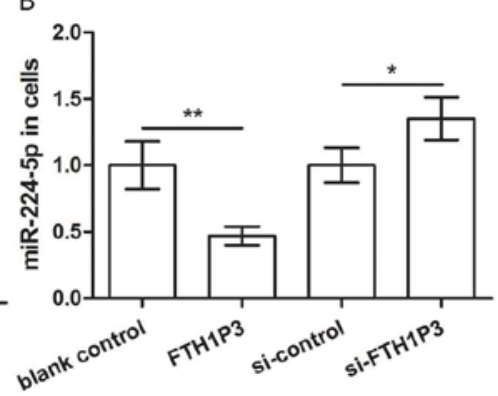

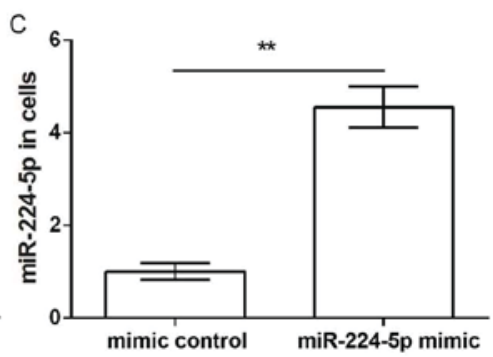

Figure 2. IncRNA FTH1P3 inhibits miR-224-5p expression. (A) miR-224-5p expression in low- and high-grade glioma tissues in addition to adjacent normal tissues. (B) miR-224-5p expression in glioma U251 cells transfected with pc-FTH1P3, si-FTH1P3 and the corresponding controls. (C) The expression of miR-224-5p in glioma U251 cells transfected with miR-224-5p mimic and the corresponding control. ${ }^{*} \mathrm{P}<0.05,{ }^{* *} \mathrm{P}<0.01$. lncRNA, long non-coding RNA; FTH1P3, ferritin heavy polypeptide 1 pseudogene 3; pc, pcDNA3.1; si, small interfering RNA; miR-224-5p, microRNA-224-5p; NS, not significant.

A

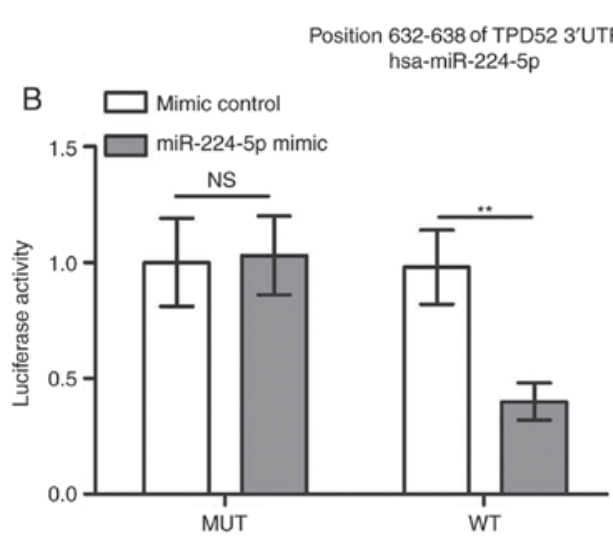

D

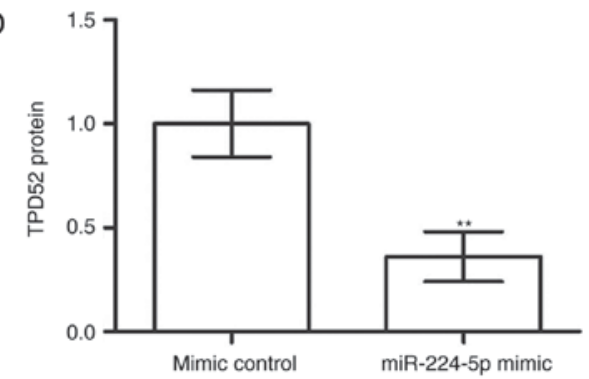

Predicted consequential pairing of target region (top) and miRNA (bottom)

5'- AAUCAAACUUCUAG GUGACUU G

3'-UUGCCUUGGUGAU- CAUGAA C

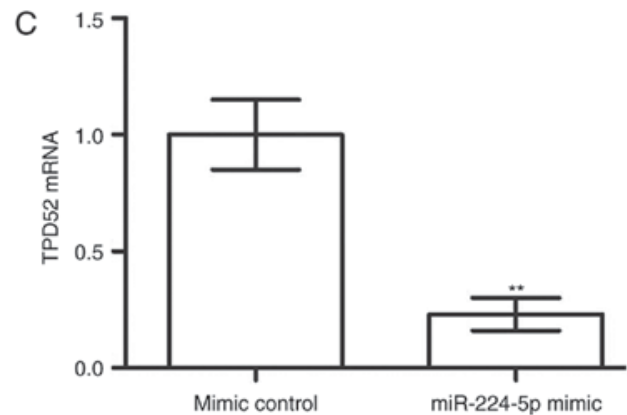

TPD52

GAPDH

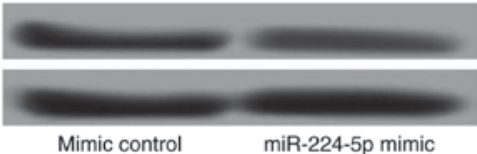

Figure 3. TPD52 is a target of miR-224-5p. (A) TargetScanHuman was used to predict the binding sequence between TPD52 and miR-224-5p. (B) Luciferase activity of TPD52 3'-UTR-WT and TPD52 3'-UTR-MUT in the presence of miR-224-5p mimic or mimic control. (C) mRNA and (D) protein expression of TPD52 in U251 cells transfected with miR-224-5p mimic or mimic control. ${ }^{* *} \mathrm{P}<0.05$ vs. mimic control. TPD52, tumor protein D52; miR-224-5p, microRNA-224-5p; MUT, mutant, WT, wild-type; 3'UTR, 3'untranslated region; NS, not significant.

examine the functional role of miR-224-5p in glioma U251 cells, miR-224-5p was overexpressed. As presented in Fig. 2C, the results demonstrated that the expression of miR-224-5p was significantly increased by transfection with miR-224-5p mimic, indicating a high transfection efficiency $(\mathrm{P}<0.01)$.

miR-244-5p negatively regulates TPD52 expression. To further examine the downstream regulatory mechanism of miR-224-5p, the target genes of miR-224-5p were predicted by TargetScanHuman. The results revealed that TPD52 was a potential target of miR-224-5p (Fig. 3A). The luciferase reporter assay demonstrated that miR-224-5p mimic decreased the luciferase activity of TPD52 3'-UTR-WT $(\mathrm{P}<0.05)$, although not that of TPD52 3'-UTR-MUT (Fig. 3B). In addition, the
mRNA and protein expression of TPD52 was significantly decreased following overexpression of miR-224-5p $(\mathrm{P}<0.05$; Fig. 3C and D). These data indicated that TPD52 was a target of miR-224-5p, and TPD52 expression was negatively regulated by $\operatorname{miR}-224-5 p$.

FTH1P3 may exert its effects in glioma via the miR-224-5p/TPD52 axis. The expression of TPD52 in glioma tissues was further determined by western blot analysis. The results revealed that TPD52 expression in glioma tissues was significantly increased compared with normal tissues, with the highest expression in high-grade glioma tissues $(\mathrm{P}<0.05$; Fig. 4A). To further analyze the functional role of TPD52, U251 cells were transfected with pcDNA-TPD52. The results 
A
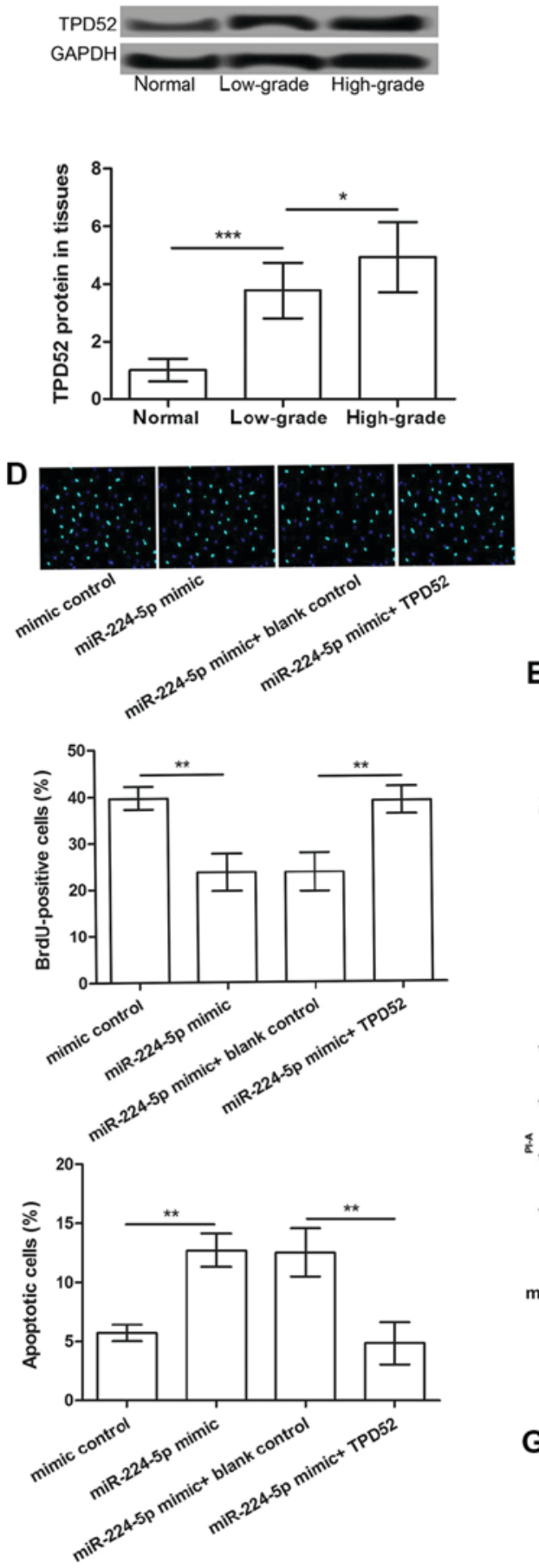

G
B
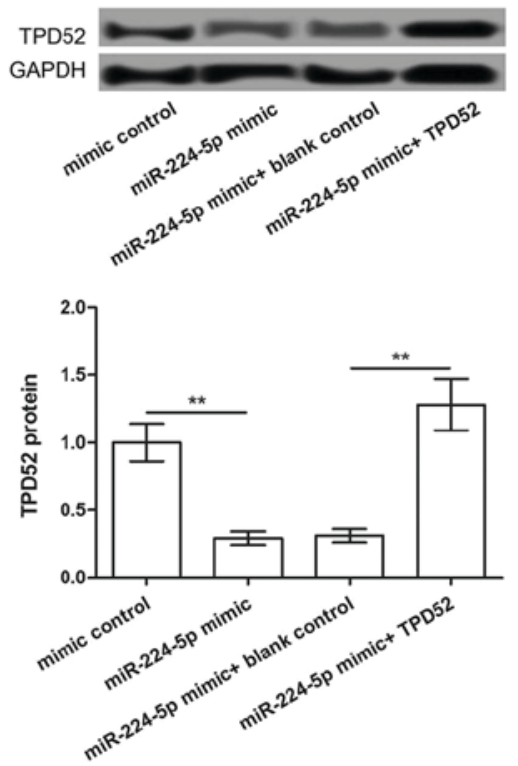

$\mathbf{E}$
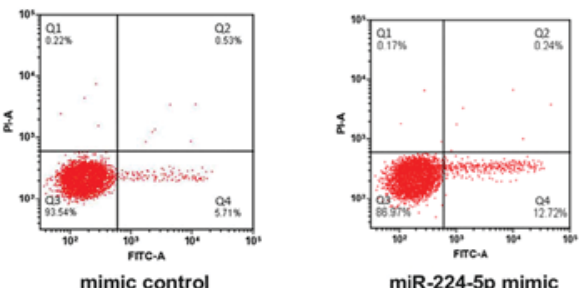

miR-224-5p mimic
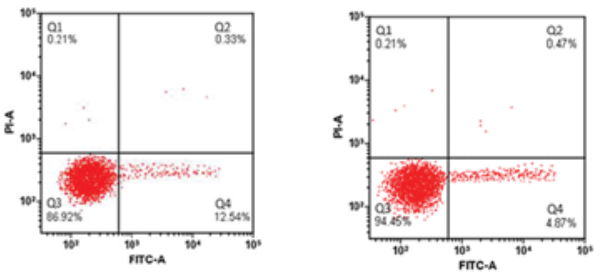

miR-224-5p mimic+ blank control

miR-224-5p mimic+ TPD52
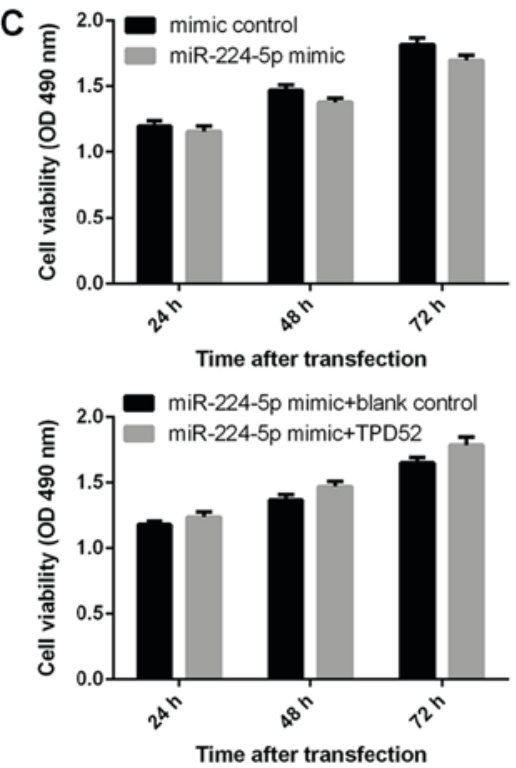

$\mathbf{F}$
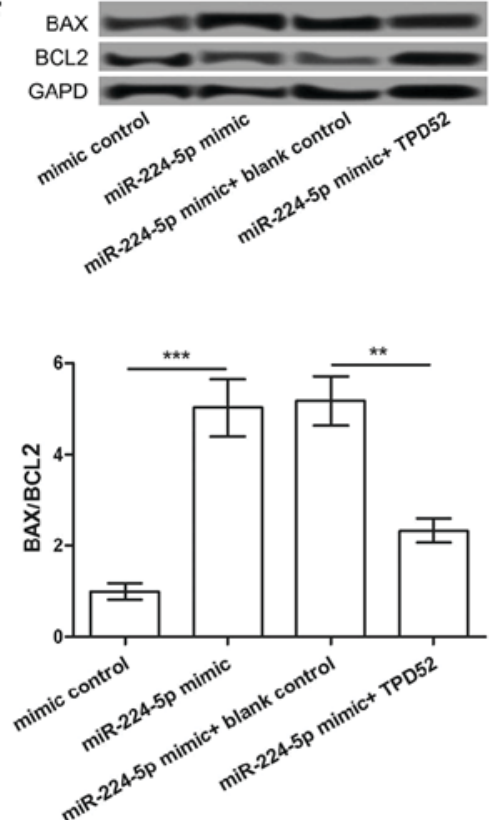

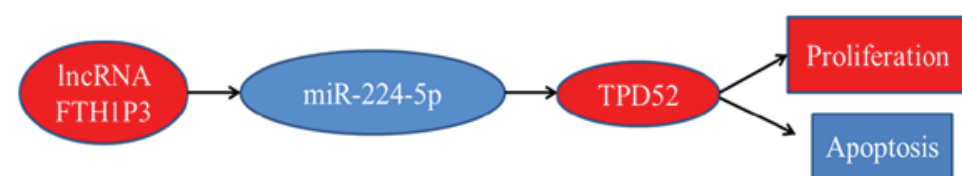

Figure 4. The miR-224-5p/TPD52 axis may be a functional mechanism of FTH1P3 in glioma. (A) TPD52 expression in low- and high-grade glioma tissues and adjacent normal tissues. (B) The expression of TPD52 in glioma U251 cells treated with mimic control, miR-224-5p mimic, miR-224-5p mimic + blank control, miR-224-5p mimic + pc-TPD52. (C) The cell viability of each group after 24, 48 and $72 \mathrm{~h}$ was determined by MTT assay. (D) A BrdU incorporation assay showed BrdU-positive cells in different transfected groups (magnification, $\mathrm{x} 400$ ). (E) The percentage of apoptotic cells in each group was detected by flow cytometry. (F) Western blot analysis showed the expression levels of BAX/BCL2 in different transfected groups. (G) Schematic representation of the potential mechanism of action of IncRNA FTH1P23 in glioma. ${ }^{*} \mathrm{P}<0.05,{ }^{* * *} \mathrm{P}<0.01$ and ${ }^{* * *} \mathrm{P}<0.001$. IncRNA, long non-coding RNA; FTH1P3, ferritin heavy polypeptide 1 pseudogene 3; BrdU, 5-bromo-2-deoxyuridine; BAX, apoptosis regulator BAX; BCL2, B cell lymphoma 2; TPD52, tumor protein D52; miR-224-5p, microRNA-224-5p.

demonstrated that the protein expression of TPD52 was significantly increased by pcDNA-TPD52 ( $\mathrm{P}<0.001$; Fig. 4B). Additionally, compared with the mimic control group, TPD52 expression was significantly downregulated in U251 cells following transfection with miR-224-5p mimic. This effect was markedly reversed following co-transfection of miR-224-5p mimic and pc-TPD52 (P<0.01; Fig. 4C). Furthermore, the results of the MTT and BrdU assays revealed that cell viability and the number of BrdU-positive cells was significantly inhibited following miR-224-5p overexpression. These effects were also markedly reversed following co-transfection with miR-224-5p mimic and pc-TPD52 ( $<<0.05$, at $24 \mathrm{~h} ; \mathrm{P}<0.01$, at 
$48 \mathrm{~h}$ and $\mathrm{P}<0.01$ at $72 \mathrm{~h}$; Fig. 4D and E). Finally, miR-224-5p mimic transfection significantly induced cellular apoptosis and increased the expression of BAX/BCL2 in U251 cells, which was significantly reversed following co-transfection of miR-224-5p and TPD52 ( $\mathrm{P}<0.01$; Fig. $4 \mathrm{~F}$ and $\mathrm{P}<0.001$ miR-224-5p mimic vs. mimic control, $\mathrm{P}<0.01$, miR-224-5p mimic+TPD52 vs. miR-224-5p mimic + blank control; Fig. 4G). Taken together, these data indicated that FTH1P3 may act through the miR-224-5p/TPD52 axis in glioma (Fig. 4H).

\section{Discussion}

lncRNAs have emerged as key regulators in cancer biology (9). In the present study, the role and regulatory mechanism of lncRNA FTH1P3 in glioma was investigated. The results revealed that FTH1P3 was upregulated in glioma tissues, and its upregulation promoted glioma cell proliferation and inhibited apoptosis. In addition, FTH1P3 inhibited miR-224-5p expression, which in turn negatively regulated TPD52 expression. Thus, the miR-224-5p/TPD52 axis may be a downstream mechanism by which FTH1P3 may regulate glioma cell proliferation and apoptosis. These findings suggested that the FTH1P3/miR-224-5p/TPD52 axis may have a key role in glioma progression.

Aberrant expression of miR-224 has been associated with the development of numerous cancer types. Xia et al (20) demonstrated that the upregulation of miR-224 promotes cell proliferation and migration in gastric cancer (20). Zhang et al (21) confirmed that miR-224 is upregulated in colorectal cancer and promotes cell proliferation and invasion. In addition, dysregulation of miR-224-5p is correlated with cisplatin resistance in ovarian papillary serous carcinoma (22). Notably, upregulation of miR-224 is also correlated with poor prognosis in glioma patients (23). In the present study, FTH1P3 was overexpressed and its upregulation is correlated to glioma cell proliferation and apoptosis. FTH1P3 inhibited miR-224-5p expression, and overexpression of miR-224-5p inhibited U251 cell proliferation and induced cellular apoptosis. Given the key role of miR-224-5p in numerous types of cancer, it was speculated that upregulation of FTH1P3 may promote cell proliferation and inhibit apoptosis in glioma via negative regulation of miR-224-5p expression.

Furthermore, TPD52 was identified as a target of miR-224-5p in the current study. Nearly 20 years ago, TPD52 was originally identified as an overexpressed gene in human breast cancer (24). Accumulating evidence has confirmed that TPD52 contributes to the development and progression of various human cancer types (25-28). Decreased TPD52 expression is correlated with poor prognosis in primary hepatocellular carcinoma (29). Additionally, miR-218 may function as a tumor suppressor in lung squamous cell carcinoma by targeting TPD52 (30). Li et al (31) demonstrated that miR-34a inhibits the metastasis of breast cancer by targeting oncogenic TPD52. Notably, miR-224 suppresses the migration and invasion of prostate cancer cells via TPD52 inhibition (18). In the present study, FTH1P3 inhibited miR-224-5p expression, which negatively regulated TPD52 expression. Overexpression of miR-224-5p significantly inhibited U251 cell proliferation and induced cellular apoptosis; this effect was clearly reversed following co-transfection of miR-224-5p and TPD52. Taken together, these findings indicated that miR-224-5p may control the proliferation and apoptosis of glioma cells via TPD52 inhibition, and FTH1P3 may exert its effects on glioma cells via the miR-224-5p/TPD52 axis.

In conclusion, the data revealed that upregulation of FTH1P3 may promote glioma cell proliferation and inhibit apoptosis via regulation of the miR-224-5p/TPD52 axis. FTH1P3 may provide a novel perspective on the pathogenesis of glioma. Future experiments aiming at exploring the possible regulatory mechanism of FTH1P3 in the other kinds of glioma cells are required in order to further investigate the findings of the present study. The findings of the present study may aid the provision of a theoretical basis for the clinical therapy of glioma.

\section{Acknowledgements}

Not applicable.

\section{Funding}

Not applicable.

\section{Availability of data and materials}

The datasets used and/or analyzed during the current study are available from the corresponding author on reasonable request.

\section{Authors' contributions}

YZ designed this study, wrote the manuscript and performed the MTT assay, RNA pull-down assay, western blotting and RT-qPCR; YL performed the cell apoptosis analysis, JW contributed the data analysis and PL helped to collect data.

\section{Ethics approval and consent to participate}

The present study was approved by the ethics committee of Tianjin Medical University General Hospital according to the criteria of the Declaration of Helsinki. Informed consent was acquired from each patient.

\section{Patient consent for publication}

Not applicable.

\section{Competing interests}

The authors declare that they have no competing interests.

\section{References}

1. Omuro A and DeAngelis LM: Glioblastoma and other malignant gliomas: A clinical review. Jama 310: 1842-1850, 2013.

2. Gallego O: Nonsurgical treatment of recurrent glioblastoma. Curr Oncol 22: e273-e281, 2015.

3. Rao JS: Molecular mechanisms of glioma invasiveness: The role of proteases. Nat Rev Cancer 3: 489-501, 2003.

4. Shi X, Sun M, Liu H, Yao Y and Song Y: Long non-coding RNAs: A new frontier in the study of human diseases. Cancer Lett 339: 159-166, 2013. 
5. Iyer MK, Niknafs YS, Malik R, Singhal U, Sahu A, Hosono Y, Barrette TR, Prensner JR, Evans JR, Zhao S, et al: The landscape of long noncoding RNAs in the human transcriptome. Nat Genet 47: 199-208, 2015.

6. Boon RA, Jaé N, Holdt L and Dimmeler S: Long Noncoding RNAs: From Clinical Genetics to Therapeutic Targets. J Am Coll Cardiol 67: 1214-1226, 2016.

7. Chen X, Fan S and Song E: Noncoding RNAs: New players in cancers. Adv Exp Med Biol 927: 1-14, 2016.

8. Martens-Uzunova ES, Böttcher R, Croce CM, Jenster G, Visakorpi T and Calin GA: Long noncoding RNA in prostate, bladder and kidney cancer. Eur Urol 65: 1140-1151, 2014.

9. Huarte M: The emerging role of lncRNAs in cancer. Nat Med 21: $1253-1261,2015$

10. Yan X, Hu Z, Feng Y, Hu X, Yuan J, Zhao SD, Zhang Y, Yang L, Shan W, He Q, et al: Comprehensive genomic characterization of long non-coding RNAs across human cancers. Cancer Cell 28: 529-540, 2015

11. Fatima R, Akhade VS, Pal D and Rao SM: Long noncoding RNAs in development and cancer: Potential biomarkers and therapeutic targets. Mol Cell Ther 3: 5, 2015.

12. Ma KX, Wang HJ, Li XR, Li T, Su G, Yang P and Wu JW: Long noncoding RNA MALAT1 associates with the malignant status and poor prognosis in glioma. Tumor Biol 36: 3355-3359, 2015.

13. Wang Q, Zhang J, Liu Y, Zhang W, Zhou J, Duan R, Pu P, Kang C and Han L: A novel cell cycle-associated lncRNA, HOXA11-AS is transcribed from the 5-prime end of the HOXA transcript and is a biomarker of progression in glioma. Cancer Lett 373: 251-259, 2016

14. Yao Y, Ma J, Xue Y, Wang P, Li Z, Liu J, Chen L, Xi Z, Teng H, Wang Z, et al: Knockdown of long non-coding RNA XIST exerts tumor-suppressive functions in human glioblastoma stem cells by up-regulating miR-152. Cancer Lett 359: 75-86, 2015.

15. Jiang X, Yan Y, Hu M, Chen X, Wang Y, Dai Y, Wu D, Wang Y, Zhuang Z and Xia H: Increased level of H19 long noncoding RNA promotes invasion, angiogenesis and stemness of glioblastoma cells. J Neurosurg 124: 129-136, 2016.

16. Zhang CZ: Long non-coding RNA FTH1P3 facilitates oral squamous cell carcinoma progression by acting as a molecular sponge of miR-224-5p to modulate fizzled 5 expression. Gene 607: 47-55, 2017.

17. Zheng X, Tang H, Zhao X, Sun Y, Jiang Y and Liu Y: Long non-coding RNA FTH1P3 facilitates uveal melanoma cell growth and invasion through miR-224-5p. PLoS One 12: e0184746, 2017.

18. Goto Y, Nishikawa R, Kojima S, Chiyomaru T, Enokida H, Inoguchi S, Kinoshita T, Fuse M, Sakamoto S, Nakagawa M, et al: Tumour-suppressive microRNA-224 inhibits cancer cell migration and invasion via targeting oncogenic TPD52 in prostate cancer. Febs Lett 588: 1973-1982, 2014.

19. Livak KJ and Schmittgen TD: Analysis of relative gene expression data using real-time quantitative PCR and the 2(-Delta Delta C(T)) method. Methods 25: 402-408, 2001.
20. Xia M, Wei J and Tong K: MiR-224 promotes proliferation and migration of gastric cancer cells through targeting PAK4. Pharmazie 71: 460-464, 2016.

21. Zhang GJ, Zhou H, Xiao H, Li Y and Zhou T: Up-regulation of miR-224 promotes cancer cell proliferation and invasion and predicts relapse of colorectal cancer. Cancer Cell Int 13: 104, 2013.

22. Zhao H, Bi T, Qu Z, Jiang J, Cui S and Wang Y: Expression of miR-224-5p is associated with the original cisplatin resistance of ovarian papillary serous carcinoma. Oncol Rep 32: 1003-1012, 2014.

23. Lu S, Wang S, Geng S, Ma S, Liang Z and Jiao B: Upregulation of microRNA-224 confers a poor prognosis in glioma patients. Clin Transl Oncol 15: 569-574, 2013.

24. Byrne JA, Tomasetto C, Garnier JM, Rouyer N, Mattei MG, Bellocq JP, Rio MC and Basset P: A screening method to identify genes commonly overexpressed in carcinomas and the identification of a novel complementary DNA sequence. Cancer Res 55: 2896-2903, 1995.

25. Byrne JA, Frost S, Chen Y and Bright RK: Tumor protein D52 (TPD52) and cancer-oncogene understudy or understudied oncogene? Tumor Biol 35: 7369-7382, 2014.

26. Moritz T, Venz S, Junker H, Kreuz S, Walther $\mathrm{R}$ and Zimmermann U: Isoform 1 of TPD52 (PC-1) promotes neuroendocrine transdifferentiation in prostate cancer cells. Tumor Biol 37: 10435-10446, 2016.

27. Ummanni R, Teller S, Junker H, Zimmermann U, Venz S, Scharf C, Giebel J and Walther R: Altered expression of tumor protein D52 regulates apoptosis and migration of prostate cancer cells. FEBS J 275: 5703-5713, 2008.

28. Zhao Z, Liu H, Hou J, Li T, Du X, Zhao X, Xu W, Xu W and Chang J: Tumor protein D52 (TPD52) inhibits growth and metastasis in renal cell carcinoma cells through the PI3K/Akt signaling pathway. Oncol Res 25: 773-779, 2017.

29. Wang Y, Chen CL, Pan QZ, Wu YY, Zhao JJ, Jiang SS, Chao J, Zhang XF, Zhang HX, Zhou ZQ, et al: Decreased TPD52 expression is associated with poor prognosis in primary hepatocellular carcinoma. Oncotarget 7: 6323, 2016.

30. Kumamoto T, Seki N, Mataki H, et al: Tumor-Suppressive MicroRNA-218 Inhibits Cancer Cell Migration And Invasion Directly Targeting TPD52 In Lung Squamous Cell Carcinoma. In: A71. Oncogenes and Angiogenesis In Lung Tumors. Am Thoracic Soc, ppA2371, 2017.

31. Li G, Yao L, Zhang J, Li X, Dang S, Zeng K, Zhou Y and Gao F: Tumor-suppressive microRNA-34a inhibits breast cancer cell migration and invasion via targeting oncogenic TPD52. Tumor Biol 37: 7481-7491, 2016.

This work is licensed under a Creative Commons Attribution-NonCommercial-NoDerivatives 4.0 International (CC BY-NC-ND 4.0) License. 